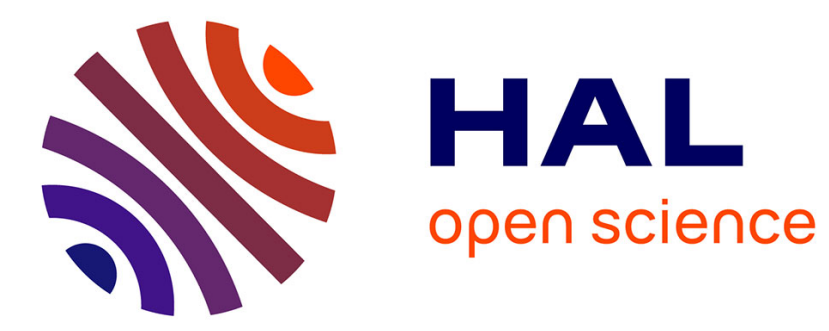

\title{
La réflectivité de neutrons polarisés avec analyse de polarisation: un outil de choix pour l'étude des couches minces magnétiques
}

F. Ott

\section{- To cite this version:}

F. Ott. La réflectivité de neutrons polarisés avec analyse de polarisation : un outil de choix pour l'étude des couches minces magnétiques. Journal de Physique IV Proceedings, 1996, 06 (C7), pp.C7-77-C7-81. 10.1051/jp4:1996709 . jpa-00254497

\section{HAL Id: jpa-00254497 https://hal.science/jpa-00254497}

Submitted on 1 Jan 1996

HAL is a multi-disciplinary open access archive for the deposit and dissemination of scientific research documents, whether they are published or not. The documents may come from teaching and research institutions in France or abroad, or from public or private research centers.
L'archive ouverte pluridisciplinaire HAL, est destinée au dépôt et à la diffusion de documents scientifiques de niveau recherche, publiés ou non, émanant des établissements d'enseignement et de recherche français ou étrangers, des laboratoires publics ou privés. 


\section{La réflectivité de neutrons polarisés avec analyse de polarisation : un outil de choix pour l'étude des couches minces magnétiques}

F. Ott

Laboratoire Léon Brillouin, Centre d'Études de Saclay, 91191 Gif-sur-Yvette cedex, France

Résumé : La réllectivité de neutrons polariscés avec analyse de polarisation est une technique qui permet d'ohlenir des renseignements très précicux sur la structure de couches minces magnétiques. Outre la détermination de la structure chimique des films (épaisseur, interdiffusion entre couches...) comme le permettent les techniques classiques de réflectivité, la réflectivité de neutrons polarisés permet de déterminer des profils d'aimantation vectoriels dans des films ou des multicouches magnétiques.

\section{INTRODUCTION}

Ces dernières années ont vu un grand regain dans l'étude des couches minces magnétiques. De nouveaux phénomènes physiques ont été observés dans ces structures artificielles nanométriques. En effet à ces échelles que l'on maîtrise bien maintenant, les propriétés de surface jouent un rôle prépondérant. On voit donc l'apparition de phénomènes tels que l'anisotropie magnétique perpendiculaire ou bien la diminution ou l'augmentation des moments en surface. La réflectométrie de neutrons polarisés a subi un grand essor depuis une dizaine d'années. C'est un outil pratiquement unique pour l'étude de ces problèmes. En effet, la réflectivité de neutrons avec analyse de polarisation permet de déterminer un profil d'aimantation vectoriel dans l'épaisseur d'un film magnétique.

\section{LA REFLECTIVITE DE NEUTRONS}

La réflectivité de neutrons est une technique identique à la réflectivité de rayons $X$ [1]. En mesurant la refflexion spéculaire d'un faisceau de neutrons en fonction de l'angle d'incidence, on peut déterminer la structure interne d'un film mince. L'amplitude de diffusion magnétique des neutrons étant du même ordre de grandeur que l'amplitude de diffusion nucléaire, l'utilisation de neutrons polarisés permet de déterminer des profils d'aimantation vectoriels dans des structures assez complexes [2-4]. Si on considère un faisceau de neutrons ayant deux états de polarisation up et down, les courbes de reflectivité des neutrons conservant leur état de polarisation après réflexion (incident up (resp. down)), réfléchis up (resp. down)) donnent des informations sur l'aimantation le long de l'axe de quantification des spins des neutrons (i.e. le champ extérieur appliqué). Les courbes de réflectivité des neutrons ayant flippé après réflexion sur l'échantillon (incident up (resp. down), réfléchis down (resp. up)) donnent des informations sur l'aimantation perpendiculaire à l'axe de quantification. Un formalisme complet peut être trouvé dans la référence [5]. 


\section{LE REFLECTOMETRE, A NEUTRONS POLARISES PADA}

Le réflectomètre PADA construit au réacteur Orphée du Centre d'Etudes de Saclay est un spectromètre deux axes à neutrons polarisés avec analyse de polarisation. La longueur d'onde de travail est fixée à $0.4 \mathrm{~nm}$. Le flux après analyse est de $3.10^{4} \mathrm{n} / \mathrm{s} / \mathrm{cm}^{2}$. La divergence du faisceau est réduite à $0.03^{\circ}$. La polarisation et l'analyse sont réalisées par l'utilisation de supermiroirs polarisants. Pour augmenter le rapport de flipping, on effectue une double réflexion dans le polariseur et l'analyseur. La transmission de chaque polariseur est d'environ $70 \%$. On peut obtenir des rapports de flipping allant jusqu'à 80 . Un cryostat contenant deux bobines supraconductrices permet de faire varier la température de $1.5 \mathrm{~K}$ à $300 \mathrm{~K}$ dans un champ de 3.5 T appliqué soit parallèlement soit perpendiculairement au plan de l'échantillon. La figure 1 montre un schéma du dispositit expérimental.

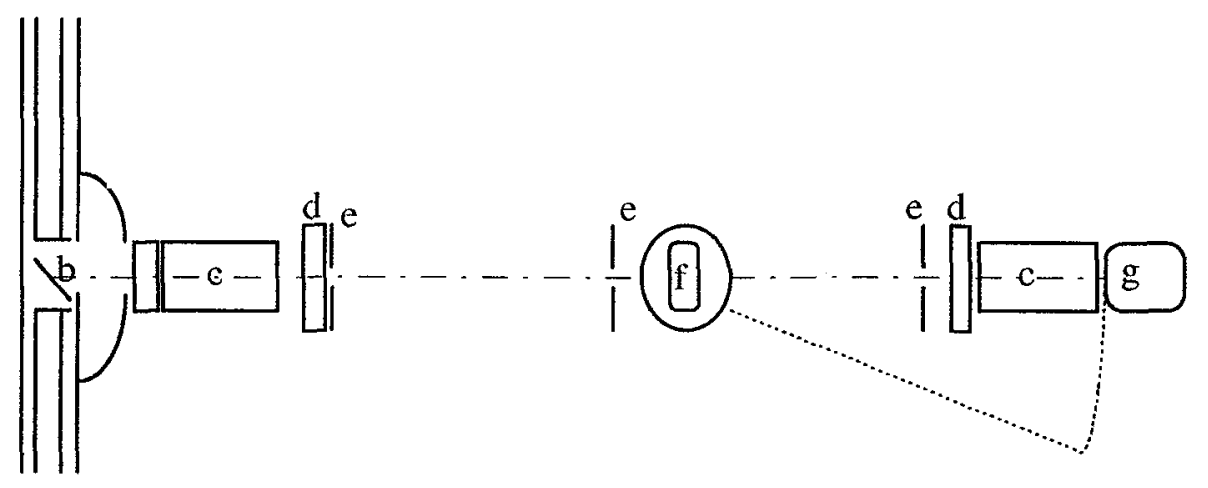

Figure 1: Un monocristal de griphlite (b) permet d'oltenir un faisceall monochromatique $(\lambda=4 \AA)$. On polarise les neutrons par une rifferion sur un supermiroir polarisant (c). On peut changer le signe de polarisation du faisceau grace aur "flippers" (d). Le faisceau es fortement collimaté par les fentes (e). Après

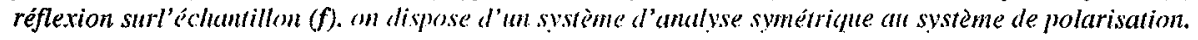

\section{POSSIBILITES DE MESURES}

La technique de réflectivití de neutrons polarisés permet de faire des mesures sur des structures en multicouches et de déterminer directement l'ordre magnétique : ferromagnétique, antiferromagnétique. hélicoïdal... Des mesures très intéressantes sont présentées dans un article de C. Dufour sur la diffraction de neutrons par les superréseaux et films épitaxiés à base de terres rares [6]. Il est aussi possible de fuire des mesures de cycles d'hystérésis sélectifs en profondeur [7]. Ces mesures donnent des valeurs absolues en $\mu_{3}$ par atome.

De manière générale, la sensibilité de la technique de réflectivité de neutrons polarisés permet de mesurer des moments inférieurs à $0.1 \mu \mathrm{B}$ par atome. Les moments de très fines couches de fer (2-3 monocouches) en sandwich entre deux couches de tungstène ont pu être mesurés [8]. Sur des échantillons plus épais tels que des monocristaux de fer la précision peut atteindre $0.01 \mu \mathrm{B}$. 


\section{EXEMPLES DE MESURES}

L'apport essentiel de la technique de réflectivité de neutrons polarisés avec analyse de polarisation est la mesure de profils magnétiques vectoriels. Dans des couches simples, des rotations de l'aimantation peuvent apparaître sous l'effet d'anisotropies de surface [9], de polarisation RKKY de surfuce [10] ou de contraintes mécaniques [11].

\subsection{Profil d'aimantation dans une tricouche $A u(8 n m) / \operatorname{Co}(3 n m) / A u(28 n m)[9]$.}

Pour illustrer la sensihilité de la technique, citons l'exemple d'une tricouche $\mathrm{Au}(8 \mathrm{~nm}) / \mathrm{Co}(3 \mathrm{~nm}) / \mathrm{Au}(2 \mathrm{nnm})$ déposée sur float glass. Les mesures de neutrons montrent que l'aimantation n'est pas homogène clans toute l'épaisseur de la couche de cobalt mais part d'une direction planaire au niveill de fit première couche d'or qui est parfaitement plane et sort progressivement du plan pout atteindre un angle de $15^{\circ}$ au niveau de la deuxième couche d'or qui est plus rugueuse (cf. figure 2)

\begin{tabular}{|c|c|}
\hline Inlerlace (Air/Au) & $1.6 \mathrm{~nm}$ \\
\hline \multicolumn{2}{|l|}{ Au $(111)$} \\
\hline \multicolumn{2}{|l|}{ Interface $(\mathrm{Co} / \mathrm{Au})$} \\
\hline \multicolumn{2}{|l|}{......... Co hep $(0001)$} \\
\hline \multicolumn{2}{|l|}{ Interface ( $\mathrm{Co} / \mathrm{Au})$} \\
\hline \multicolumn{2}{|l|}{ All (111) } \\
\hline Interlace (Au/float & $<1.4 \mathrm{~nm}$ \\
\hline
\end{tabular}

Float glass

Figure 2 : description de la triconche et résultat de l'ajustement numérique des courbes mesurées par réflectivité de neutrons. Le's alliurges d'interfucé omt une composition fivée à $50 \%$ de chaque élément.

\subsection{Profil d'aimantation dans des couches minces de nickel soumises à des contraintes mécaniques [11].}

Dans des films minces de permalloy, une très forte dépendance des constantes magnétoélastique (ME) en fonction de l'épaisseur du film mince a été observée [12]. Ce comportement a été expliqué en décrivant la constante ME comme la somme d'une contribution volumique plus une contribution de surface décroissant comme l'inverse de l'épaisseur du film : $B_{\mathrm{ent}}(\mathrm{z})=$ $\mathrm{B}_{\text {bulk }}+\mathrm{B}_{\text {surd }}\left(\mathrm{z}-\mathrm{z}_{0}\right)$. Par réflectivité de neutrons polarisés, nous avons pu mettre en évidence que ce gradient de constante ME pouvait induire des gradients de rotation dans des films minces soumis à des contraintes mécaniques externes (appliquées par déformation mécanique du substrat). La figure 3 mnntre les courbes de réflectivité pour une couche de nickel de $40 \mathrm{~nm}$ non contrainte puis soumise à une contrainte d'élongation de $3 \times 10^{-4}$ (en déformation relative). Le tableau 1 résume les résultas des ajustements numériques pour deux couches différentes. L'aimantation présente des gradients d'environ $10^{\circ}$ entre la surface du film et le «bulk » du film. 


\begin{tabular}{|c|c|c|c|c|c|c|}
\hline & \multicolumn{3}{|c|}{$\begin{array}{l}\text { Is nm nickel layer deposited on } \\
\text { a silicon substrate }\end{array}$} & \multicolumn{3}{|c|}{$\begin{array}{l}\text { 40 nm nickel lalyer deposited on at } \\
\text { glass substrate }\end{array}$} \\
\hline & thickness $(\AA)$ & $\ln (\mu \mathrm{B})$ & $\alpha\left({ }^{\circ}\right)$ & lhickness $(\AA)$ & $\mathrm{m}(\mu \mathrm{B})$ & $\alpha\left(0^{\circ}\right)$ \\
\hline alloy layer & 26 & 0.2 & 56 & 40 & 0.177 & 62.3 \\
\hline $1^{\text {st }}$ nickel layer & 35 & $0.53 ?$ & 62 & 100 & 0.53 & 61.7 \\
\hline $2^{\text {nd }}$ nickel layer & 38 & 0.53 & 65 & 100 & 0.53 & 70 \\
\hline $3^{\text {rd }}$ nickel layer & 38 & 0.53 & 64 & 100 & 0.53 & 74.4 \\
\hline $4^{\text {th }}$ nickel layer & 35 & 0.53 & 57 & 94.5 & 0.53 & 75.3 \\
\hline surface layer & 15 & 0.37 & 41 & 31.3 & 0.5 & 56.7 \\
\hline
\end{tabular}

Tableau 1: résuné des ajustements muméricues pont des couches minces de nickel. L 'aimantation est

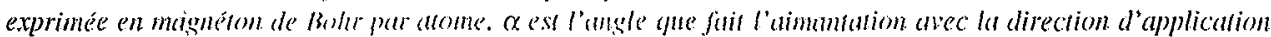

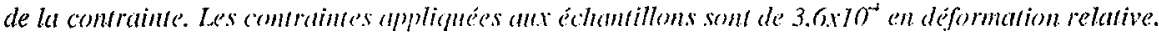
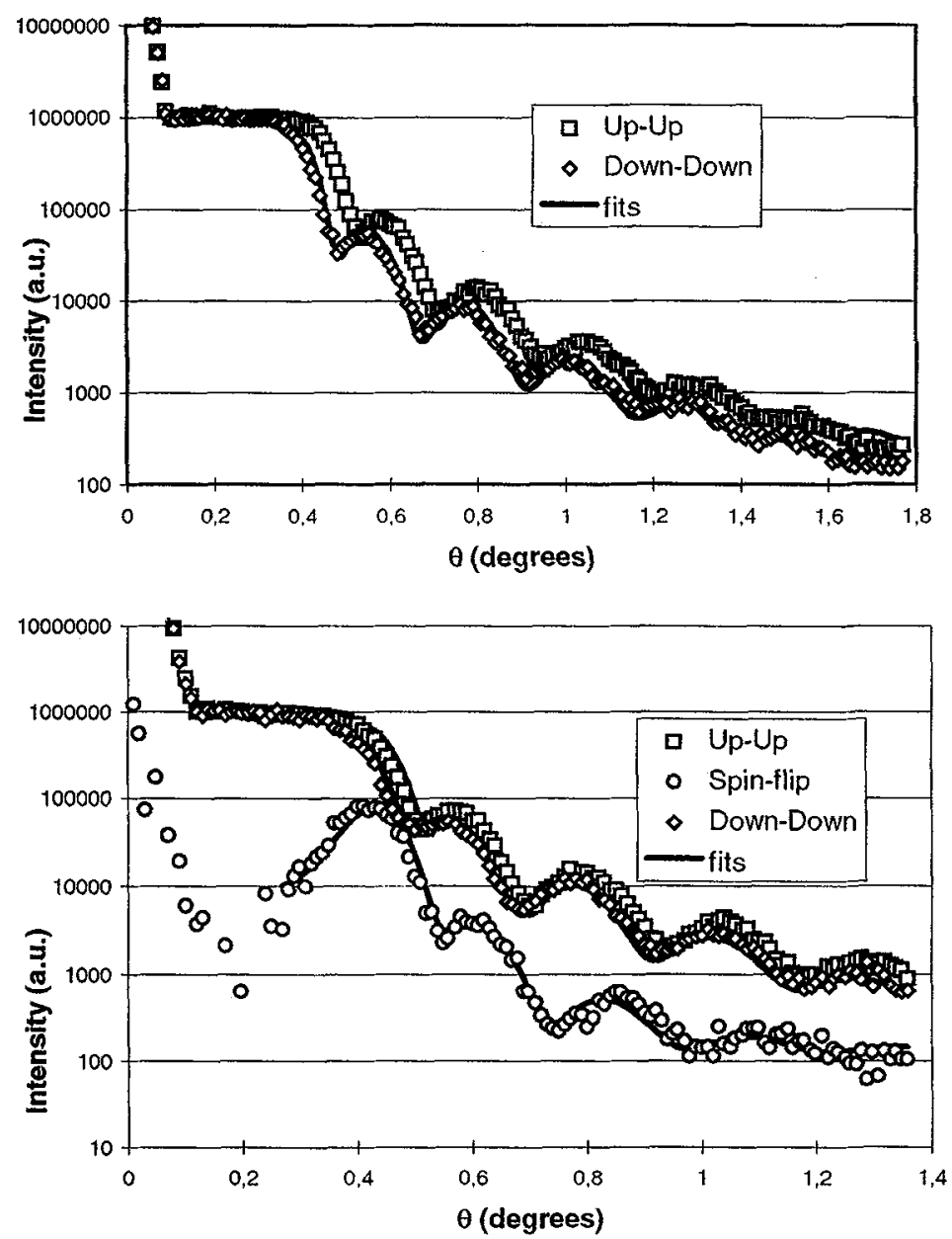

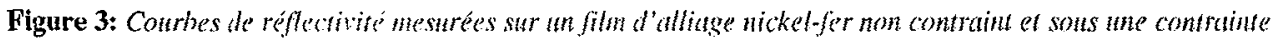

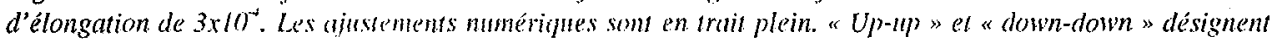
les neutrons réfléchis qui n'mu pas chungé de polarisation. "Up-down " désigne les neutrons qui ont flippé après réflexion. 


\section{CONCLUSION}

Malgré de nombreuses diflicultés expérimentales liées à la faiblesse du flux de neutrons et à la qualité des échantillons (planéité, rugosité...) et une limitation théorique importante qui est l'impossibilité de mesurer les moments perpendiculaires à la surface, la réflectivité de neutrons avec analyse de polarisition est un outil unique pour mesurer des configurations magnétiques avec une très grande sensibilité.

\section{Références}

[1] J. Lekner, Theory of reflection (Martinus Nijhotf, Dordrecht, 1987).

[2] G.P. Felcher, R. O. Hilleke, R. K. Crawford, J. Haumann R. Kleb, and G. Ostrowski, Rev. Sci. Instrum. 58 (1987) 609.

[3] N.K. Pleshamov, Z. Phys B 94, (1994) 233-243

[4] J. Blundell and J.A.C. Bland. Phys. Rev. B 46 (1992) 3391

[5] C. Fermon, Proceedings of ICNS, Sendai, Physica B 213\&214 (1995) 910-913.

[6] C. Dufour, K. Dumesnil, Ph. Mangin, G. Marchal et M. Hennion, Journal de Physique, même numéro (1997)

[7] F. Ott, C. Fermon, à paritître.

[8] O. Mc Grath, thèse de doctorat de l'université Joseph Fourier, Grenoble 1 (1994).

[9] E. Train, C. Fermon. C. Chappert, A. Megy, P. Veillet and P Beauvillain, JMMM 156 (1-3) (1996) 86-88.

[10] O.K.F. Mc Grath and al. PhysicaB 213\&214 (1995) 236-238.

[11] F. Ott and C. Fermon, to appear in J.M.M.M. (E-MRS 96 conference)

[12] O. Song, C. A. Ballentine. and R. C. O'Handley, Appl. Phys. Lett. 64, (1994) 2593. 